\title{
PA-133 WATER SUPPLY AND SANITATION CONDITIONS IN RURAL SOUTHERN MOZAMBIQUE AND ITS ASSOCIATION WITH MORBIDITY AND MORTALITY INDICATORS, 2012-2015
}

Berta Grau-Pujol, ${ }^{1,2}$ Charfudin Sacoor, ${ }^{1}$ Augusto Nhabomba, ${ }^{1}$ Aina Casellas, ${ }^{2}$ Llorenç Quintó, ${ }^{2}$ Carme Subirà, ${ }^{2}$ Ricard Giné, ${ }^{3}$ Antònia Valentín, ${ }^{2}$ Jose Muñoz ${ }^{1}$ ' CISM, Mozambique; ${ }^{2}$ ISGlobal Barcelona, Spain; ${ }^{3}$ Polytechnic University of Catalonia, Spain

\subsection{6/bmjgh-2016-000260.159}

Background Water, sanitation and hygiene (WASH) are major health determinants, responsible for an estimated world-wide disease burden of $5.7 \%$. However, the debate about the effect of water quality, hygiene and sanitation in preventing diarrhoea is still ongoing. The aim of this study is to describe access to improved water supply and sanitation infrastructure, as defined by the Joint Monitoring Programme, in the Manhiça Health Research Centre (CISM) study area and evaluate its association with morbidity and mortality indicators.

Methods We conducted a retrospective cohort study. All children under 15 living in the study area during the period 2012-2015 were included $(\mathrm{N}=61900)$. Children were followed up until they moved from the study area, turned 15 or until 2015. Water and sanitation household data were obtained from the CISM demographic surveillance system (DSS) in the Manhiça district, an area of around $2380 \mathrm{~km} 2$. Clinical data were obtained from CISM's round-the-clock morbidity surveillance system covering outpatient and hospital admissions at the Manhiça District Hospital (MDH) and rural health posts. A negative binomial regression model 
using Wald test was performed to assess the minimum community-based incidence rates (MCBIR) for every morbidity-mortality indicator.

Results Preliminary data showed that $86 \%$ of the children lived at least once in a household with unimproved sanitation facilities, $27 \%$ with an unimproved water source. Spatial distribution of unimproved water and sanitation facilities showed to be clustered. Access to unimproved sanitation and water facilities was associated to higher rates of diarrhoea, a significant $30 \%$ of diarrhoea rate increase was observed for rivers, lakes and ponds as water sources. Other morbidity indicators (malnutrition, parasitaemia, anaemia) also showed a rate increase with the use of unimproved water and sanitation facilities.

Conclusions Obtained results are useful to inform sector-related decision-making processes and ultimately improve access to safe drinking water and sanitation in rural southern Mozambique. 